\title{
Stereoselective cyclization of silylated epoxy aldehydes into piperidines. Effect of the silicon group
}

\author{
Cécile Boglio, Marie-Céline Lamas, Serge Thorimbert, *and Max Malacria* \\ Université Pierre et Marie Curie-Paris 6, Laboratoire de chimie organique (UMR CNRS 7611), \\ Institut de chimie moléculaire (FR 2769), BP 229, 4 place Jussieu, 75005 Paris, France \\ E-mail: max.malacria@upmc.fr, serge.thorimbert@upmc.fr
}

Submitted to honor the 70th anniversary of Prof. Guy Queguiner

\begin{abstract}
A series of 2- or 3-silyl-epoxy aldehydes derivatives bearing a glycinyl sidechain have been prepared and tested in intramolecular aldolization. Highly stereoselective cyclization occurs and provides the N-heterocycle framework which is useful for the synthesis of polyhydroxylated piperidines. The presence of the silicon group on the epoxy moiety strongly influenced the stereochemical outcome of the reaction.
\end{abstract}

Keywords: Silicon atom, aldolization, palladium catalysis, amination, piperidines

\section{Introduction}

Palladium plays an important role in organic chemistry due to the broad scope and versatility of its utilization in homogeneous ${ }^{1}$ or heterogeneous reactions. ${ }^{2}$ Among others, palladium-catalyzed allylation of various nucleophiles (the Tsuji-Trost reaction) is one of the major achievements in this field. However, the possibility of controlling the chemo-, regio- and enantio-selectivity of the attack of the nucleophiles onto the $\eta^{3}$-allyl ligand is still a challenging research area. ${ }^{3}$ Much work has dealt with the variation of the ligands around the metal, ${ }^{4}$ the role of the solvent, ${ }^{5}$ or the steric and electronic influence of the substituents of the allyl moiety. ${ }^{6}$ Silicon groups have been recognized as strong directing substituents. ${ }^{7}$ Organosilicon compounds are also versatile and powerful reagents leading to many applications in organic synthesis. ${ }^{8}$ They have attracted considerable attention not only as biologically acceptable analogues of natural products ${ }^{9}$ but also because silicon acts as a directing atom that increases ${ }^{10}$ or reverses selectivities usually observed for its carbon analogues. ${ }^{11}$ Since the first reports of Hiyama, organosilanes have also been extensively studied in cross-coupling reactions catalyzed by palladium. ${ }^{12}$ Some years ago, we demonstrated for the first time the highly chemo- and stereoselective alkylation of 2-silylbut-2ene-1,4-diol derivatives. ${ }^{13}$ Depending on its position, the silicon group strongly influences the 
formation and the reactivity of the cationic $\pi$-allyl palladium complex. ${ }^{14}$ The ionization takes place with complete chemoselectivity so as to expel the acetoxy group vicinal to silicon. These studies allowed the development of a new palladium-catalyzed cyclization reaction, wherein 2trialkylsilyl-1,4-diacetoxy-but-2-ene are converted, via two palladium-catalyzed reactions, into silyl-substituted cyclopentenes (Scheme 1).
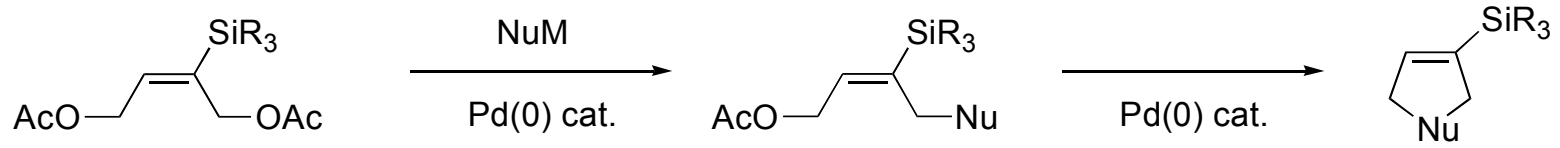

Scheme 1. Palladium-catalyzed reactions controlled by a silicon group.

In this overall annulation process the role of silicon is crucial and twofold. In fact, in the first $\mathrm{C}-\mathrm{C}$ bond formation the bulky trialkylsilyl group regiodirects the ionization of the starting bisallylic system. In addition, the same group is expected to prefer a syn disposition in the transiently generated $\eta^{3}$-allylpalladium complex, ${ }^{15}$ so as to allow the 5-endo process to take place in the second reaction. ${ }^{16}$

We also took advantage of these efficient alkylations giving stereoselectively (E) compounds, to prepare in few steps some silylated epoxy cyclopentanols. ${ }^{17}$ The high level of stereoselectivity observed during the aldolization was attributed, in part, to the presence of the three membered ring in the tether (Scheme 2).
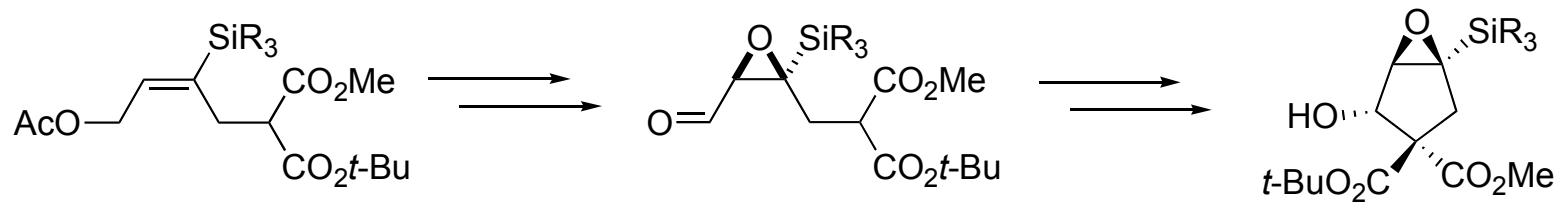

Scheme 2. Stereoselective intramolecular aldolization.

We recently extended this process towards the construction of a 6-membered nitrogen heterocycle. ${ }^{18}$ Substituted piperidines, in general, ${ }^{19}$ and polyhydroxylated derivatives ${ }^{20}$ more precisely, have been the targets of a large number of synthetic approaches ${ }^{21}$ due to their potential as therapeutic agents, and the need to find efficient access to biologically active analogues. Our synthetic approach to the piperidine skeleton is based on the stereoselective palladium-catalyzed amination as well as an intramolecular aldol condensation (Scheme 3). ${ }^{22}$
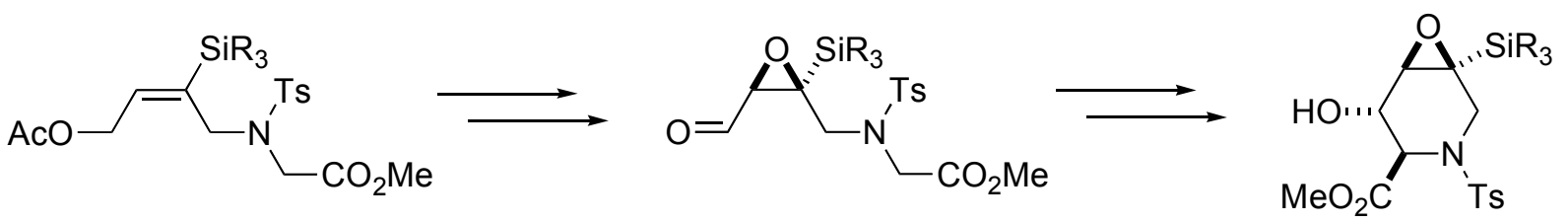

Scheme 3. Preparation of piperidines. 
We decided to study the effect of the silicon substitution on the above cyclization. In particular, we were intrigued to verify the effect of the presence of the silyl group vicinal to the carbonyl function. We report herein the preparation and the result of the cyclization of two epoxy aldehydes 1 and 2 substituted by a triethylsilyl group at the 2 or 3 position, respectively (Scheme 4).

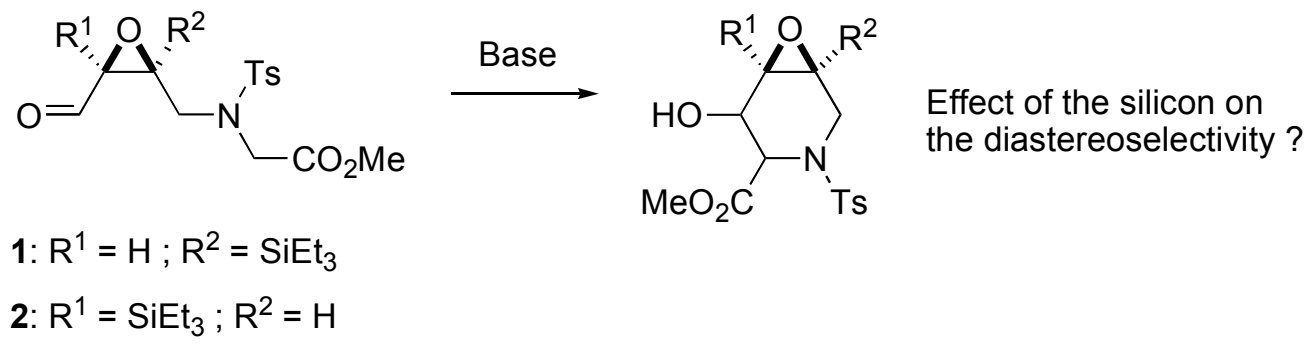

Scheme 4. Diastereoselective aldolization.

\section{Results and Discussion}

Our synthesis started from either the silyl derivative $\mathbf{3}$ bearing two allylic acetates or the corresponding dicarbonate $3^{\prime}$. As previously reported, ${ }^{18}$ the direct palladium-catalyzed chemoand stereo-selective amination gave the expected compound 4 in $50 \%$ yield with no detectable trace of the product derived from the substitution of the other acetate (Scheme 5). The same reaction using the more reactive dicarbonate $3^{\prime}$ 'delivered the expected product $4^{\prime}$ in $75 \%$ yield in a $90 / 10 \mathrm{E} / \mathrm{Z}$ ratio. Again, no product coming from the ionization of the second leaving group was detected.

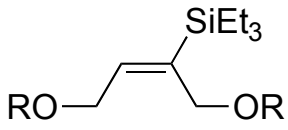

3: $\mathrm{R}=\mathrm{Ac}$

3': $\mathrm{R}=\mathrm{CO}_{2} \mathrm{Me}$

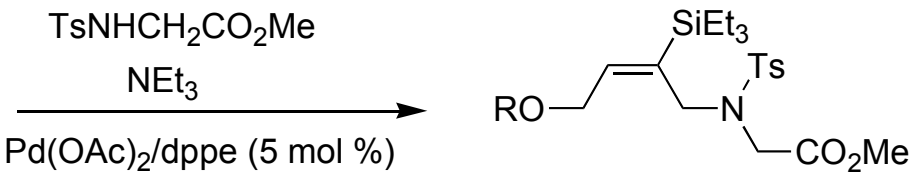

4: $R=A c ; 50 \%$

4': $\mathrm{R}=\mathrm{CO}_{2} \mathrm{Me} ; 75 \%$

Scheme 5. Chemo- and stereo-selective palladium-catalyzed amination.

To prepare the other regioisomer, some more steps were required. Indeed, 3 has two primary allylic acetates and the less hindered one needed to be deprotected. This was efficiently realized using the transesterification protocol developed by Seebach. ${ }^{23}$ One should notice that it is crucial to use a relatively hindered alcoholic solvent to perform the mild deprotection with a good chemoselectivity. Moreover, it is important to control the evolution of the reaction and to stop it when traces of the corresponding diol appear, which usually requires three hours at room 
temperature. Purification by flash chromatography allowed the isolation of the desired allylic alcohol 5 in 75\% yield (Scheme 6). After transformation of the alcohol into bromide, the reaction with the potassium salt of the N-tosyl glycine methyl ester delivered the desired allylic amino derivative 6 in $71 \%$ overall yield.

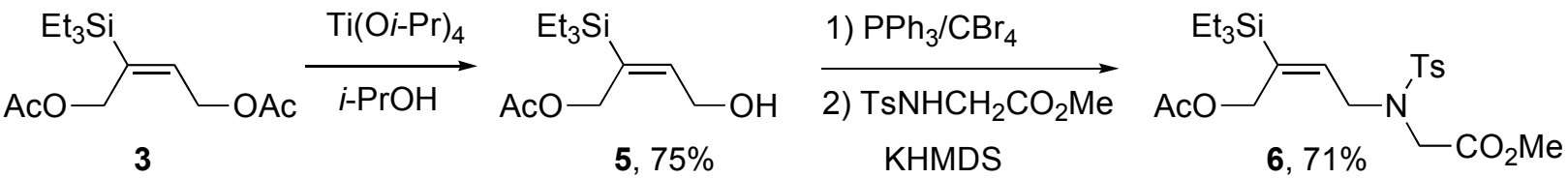

Scheme 6. Preparation of compound 6.

Having in hand the two regioisomers of the amino- allylic acetates $\mathbf{4}$ and $\mathbf{6}$, we pursued the synthesis of the targeted precursors. Methanolysis of the acetate function of $\mathbf{4}$ and $\mathbf{6}$ delivered, in good yields, the corresponding allylic alcohols $\mathbf{7}$ and $\mathbf{8}$, which were then transformed into the silylated epoxides 9 and 10 in 79 and 92\% yields, respectively (Scheme 7). The mild oxidations of the primary alcohols into the desired aldehydes bearing all the functions needed for the construction of the piperidine ring were performed with IBX in DMSO. The stable precursors 1 and 2 were both isolated in $68 \%$ yield.

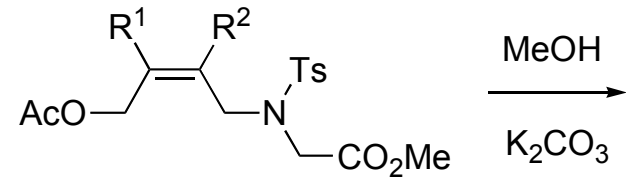

4: $R^{1}=H ; R^{2}=\mathrm{SiEt}_{3}$

6: $\mathrm{R}^{1}=\mathrm{SiEt}_{3} ; \mathrm{R}^{2}=\mathrm{H}$

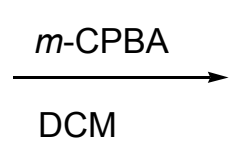

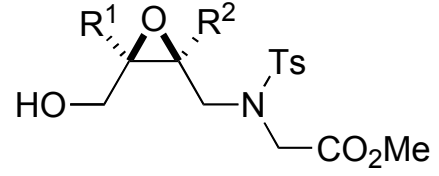

9: $\mathrm{R}^{1}=\mathrm{H} ; \mathrm{R}^{2}=\mathrm{SiEt}_{3} ; 79 \%$

10: $\mathrm{R}^{1}=\mathrm{SiEt}_{3} ; \mathrm{R}^{2}=\mathrm{H} ; 92 \%$

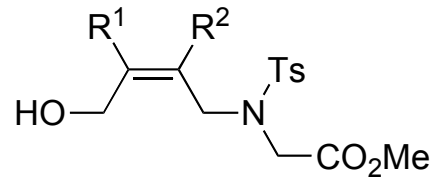

7: $\mathrm{R}^{1}=\mathrm{H} ; \mathrm{R}^{2}=\mathrm{SiEt}_{3} ; 89 \%$

8: $\mathrm{R}^{1}=\mathrm{SiEt}_{3} ; \mathrm{R}^{2}=\mathrm{H} ; 80 \%$
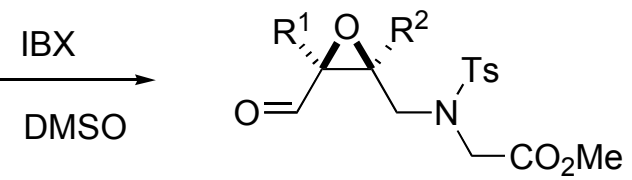

1: $\mathrm{R}^{1}=\mathrm{H} ; \mathrm{R}^{2}=\mathrm{SiEt}_{3} ; 68 \%$

2: $\mathrm{R}^{1}=\mathrm{SiEt}_{3} ; \mathrm{R}^{2}=\mathrm{H} ; 68 \%$

Scheme 7. Preparation of the silylated epoxy aldehydes $\mathbf{1}$ and $\mathbf{2}$.

We first studied the acyclic precursor 1 bearing the triethylsilyl group at the position remote from the aldehyde function. After some misleading preliminary experiments, using KHMDS, LDA or $\mathrm{NEt}_{3}$, we found that DBU in THF at room temperature was a correct base to convert 1 into the corresponding piperidine ring 11. Careful examination of the ${ }^{1} \mathrm{H}$ NMR of the crude material indicated two diastereomers (11a and 11b) in a 80/20 mixture (Scheme 8). These two 
compounds were difficult to separate by flash chromatography. However, as expected, the transrelationship between the oxirane and the created hydroxyl was totally controlled. The two diastereomers 11a and 11b correspond to the two epimers at the ester position. In the major product 11a, the ester function is trans- relative to the hydroxyl group. Attempts to improve the diastereoselectivity, by lowering the temperature considerably decrease the kinetics of the cyclization and degradation started to be competitive.

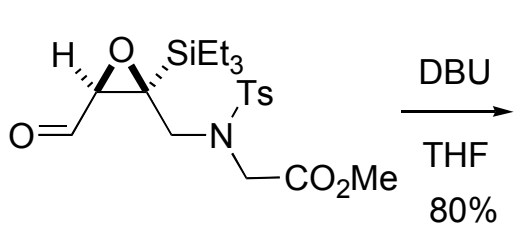

1

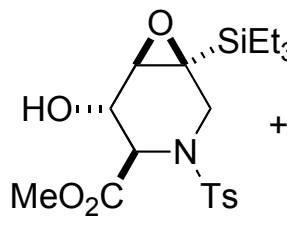

$11 \mathrm{a}$

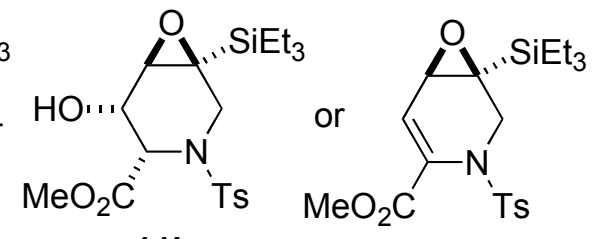

$11 b$

Scheme 8. Stereoselective preparation of piperidines.

To facilitate the purification and the isolation of the major diastereomer, we reasoned that in our basic conditions, only the minor diastereomer 11b could dehydrate into the corresponding enamino ester 12. To our pleasure, after a reaction time of 3 days, 1 gives a 75/25 mixture of 11a and the expected dehydroamino ester 12. This allowed for an easier isolation of pure 11a in $65 \%$ yield.

The second acyclic precursor $\mathbf{2}$ bearing the vicinal aldehyde and silyl functions was mixed with an equivalent amount of DBU in THF. We observed a fast consumption of the starting material and isolated after 4 hours a 1/1/0.2 mixture of three diastereomers 13 in $89 \%$ yield (Scheme 9). Attempts to separate these piperidine derivatives by flash chromatography were unsuccessful. We thus decided to apply a longer reaction time in order to favor the diastereoselective dehydration. Unfortunately, after 4 days we still isolated, in a moderate 57\% yield, a mixture of the same three diastereomers.

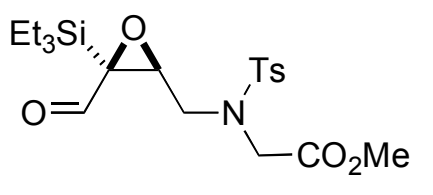

2

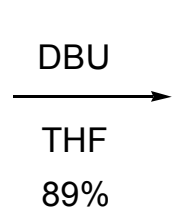

$89 \%$

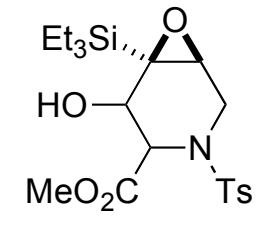

13
3 diastereomers

$1 / 1 / 0.2$ ratio

Scheme 9. Stereoselective preparation of piperidines.

Thus we can conclude that the presence or the absence of the triethylsilyl group adjacent to the aldehyde strongly influence the overall selectivity of the cyclization. Indeed, cyclization of $\mathbf{1}$ furnished only two diastereomers whereas from 2 we isolated $\mathbf{1 3}$ as a mixture of three diastereomers. We propose a model to explain the control of the relative configuration between 
the oxiranyl and the hydroxyl functions (Scheme 10). Starting from $\mathbf{1}$, where $\mathrm{R}$ is a hydrogen atom, we consider a Felkin-Ahn type model where the attack of the nucleophile occurs anti- to the electronegative group (the oxiranyl function). ${ }^{24}$ This approach should be much more favored than the approach $\mathbf{B}$ which develops electronic repulsions between the two oxygen atoms. On the other hand, starting from the molecule $2\left(\mathrm{R}=\mathrm{SiEt}_{3}\right)$, the presence of the triethylsilyl group adjacent to the carbonyl group should develop strong steric interactions in the conformation $\mathbf{A}$. Since the carbonyl group will no longer be securely syn- to a hydrogen, it could rotate to the conformation B. That might explain the loss of stereocontrol in this series and contribute to the production of the minor diastereomer of $\mathbf{1 3}$.

Oxirane/alcohol relative configuration

Ester/ alcohol relative configuration
A

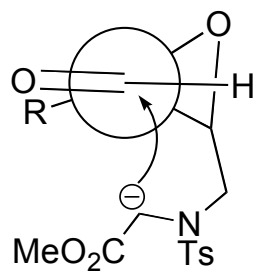

Steric interaction

C

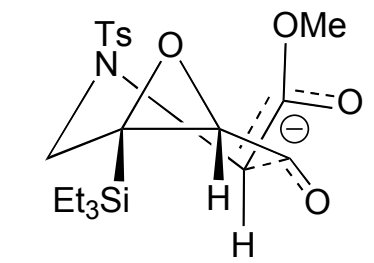

Pseudo equatorial

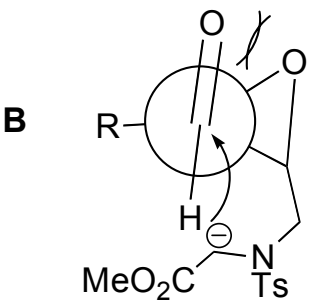

Electronic repulsion

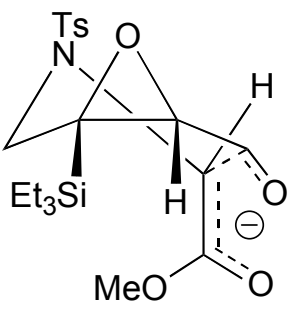

Pseudo axial

Scheme 10. Models.

To explain the major trans- relative configuration between the ester group and the hydroxyl function in the piperidine 11, we consider a chair-like transition state. Due to the pseudoequatorial position of the ester function, the approach $\mathbf{C}$ seems to be more favorable than $\mathbf{D}$. This model could explain why 11a, the anti-anti isomer, is the major isomer (11a/11b: 80/20).

Finally, we further demonstrate that the obtained piperidines could be selectively functionalized. For example, after protection of the secondary alcohol of 11a as a silyl ether, the ester group of 14 could be smoothly reduced using $\mathrm{LiAlH}_{4}$. After $1 \mathrm{~h}$ at $0^{\circ} \mathrm{C}$ in $\mathrm{Et}_{2} \mathrm{O}$, the primary alcohol 15 could be isolated in 84\% yield (Scheme 11). 


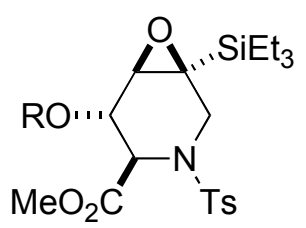

TBSCI; Im $\left(\begin{array}{c}11 \mathrm{a}: \mathrm{R}=\mathrm{H} \\ 14: \mathrm{R}=\mathrm{SiMe}_{2} t-\mathrm{Bu}\end{array}\right.$
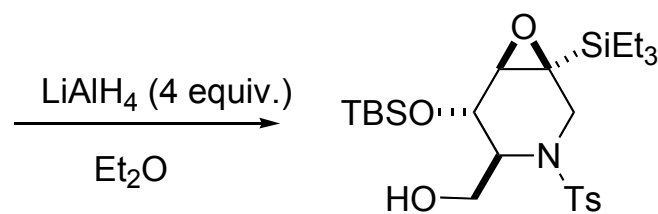

Conditions

$1 \mathrm{~h}$

$22 \mathrm{~h}$

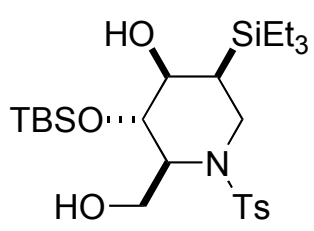

16

$84 \%$

Scheme 11. Functionalization of the piperidine ring.

On the other hand, longer reaction times allowed the clean reduction of both the ester and epoxide groups of $\mathbf{1 4}$. After $22 \mathrm{~h}$ at room temperature, we isolated the mono-protected triol 16 in $54 \%$ yield as well as traces of the corresponding detosylated analogue of 16. As expected, the reduction of the epoxysilane function is totally chemoselective and controlled by the silicon group. $^{25}$

In conclusion, we report an original access to hydroxylated piperidines. The preparation of the first acyclic precursor has been performed by a chemo- and stereo-selective palladiumcatalyzed amination of a silylated diacetate. The piperidine cycle could be obtained in a highly stereoselective aldolization. The presence or the absence of a triethylsilyl group in a neighboring position relative to the aldehyde strongly influences the overall selectivity of the cyclization. Work is underway to extend this approach to more functionalized piperidines in an enantiomeric version.

\section{Experimental Section}

General Proedures. Reagents and chemicals were purchased from commercial sources and used as received. All reactions requiring anhydrous conditions were performed under a positive pressure of argon in oven-dried glassware. All solvents were purified and distilled by standard methods. Thin layer chromatography (TLC) was performed on $0.25 \mathrm{~mm}$ E. Merck silica gel (60F-254) plates using UV light, $p$-anisaldehyde or ninhydrin. Column chromatography was carried out on Merck silica gel $60(40-63 \mu \mathrm{m})$. NMR spectra were recorded on a Bruker ARX 400. ${ }^{1} \mathrm{H}$ NMR were recorded at $400 \mathrm{MHz}$, and ${ }^{13} \mathrm{C}$ NMR at $100 \mathrm{MHz}$ with the sample solvent being $\mathrm{CDCl}_{3}$ unless otherwise noted. Chemical shifts are given in ppm, referenced to the residual proton resonances of the solvents. Coupling constants $(J)$ are given in Hertz $(\mathrm{Hz})$. The letters $\mathrm{m}$, $\mathrm{s}, \mathrm{d}, \mathrm{t}, \mathrm{q}$ mean respectively multiplet, singlet, doublet, triplet, quartet: $b r$ means that the signal is broad. IR spectra were recorded on a Bruker Tensor 27 using ATR method. Elemental analyses were carried out by the "Service de microanalyse", ICSN - CNRS, 91198 Gif sur Yvette, France or by the "Service de microanalyse", SIARE, 4 place Jussieu 75252 Paris cedex 05, France.

Compounds 3, 4 and $\mathbf{4}^{\prime}$ have already been described in refs 13b,14 and 18, respectively. 
(E)-4-Acetoxy-3-triethylsilyl-but-2-en-1-ol (5). Ti(O-i-Pr $)_{4}(23.5 \mathrm{~mL}, 78.5 \mathrm{mmol}, 1$ equiv.) was

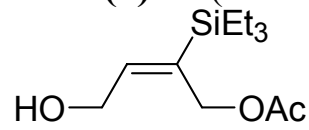

added to a solution of diacetate $3(22.5 \mathrm{~g}, 78.5 \mathrm{mmol})$ in dry $i$-PrOH under argon atmosphere. After stirring 2-3 $\mathrm{h}$ at room temperature, $\mathrm{Et}_{2} \mathrm{O}(100 \mathrm{~mL})$ and $\mathrm{HCl} 10 \%(100 \mathrm{~mL})$ were added to the mixture. The aqueous phase was extracted with $\mathrm{Et}_{2} \mathrm{O}$. The organic layer was neutralized with aqueous $\mathrm{NaHCO}_{3}$, washed with brine until neutral $\mathrm{pH}$, dried over $\mathrm{MgSO}_{4}$ and concentrated under vacuum. The yellow oil was purified on a silica gel column (PE/EA 8/2-6/4) in order to afford 5 (11.5 g, $47.1 \mathrm{mmol}, 60 \%$ yield) as a colorless oil. $\mathrm{R}_{\mathrm{f}}=0.3$ (PE/EA 75/25); IR: 3400 (large), 2940, 2860, 1740, 1610, 1230, 1010, $720 \mathrm{~cm}^{-1} ;{ }^{1} \mathrm{H}-\mathrm{NMR}\left(200 \mathrm{MHz}, \mathrm{CDCl}_{3}\right): \delta=0.64$ (q, $J=7.4 \mathrm{~Hz}$, $6 \mathrm{H}) ; 0.90(\mathrm{t}, J=7.4 \mathrm{~Hz}, 9 \mathrm{H}) ; 2.03(\mathrm{~s}, 3 \mathrm{H}) ; 4.3(\mathrm{~d}, J=6.0 \mathrm{~Hz}, 2 \mathrm{H}) ; 4.70(\mathrm{~s}, 2 \mathrm{H}) ; 6.08$ (t, $J=6.0 \mathrm{~Hz}$, $1 \mathrm{H}) ;{ }^{13} \mathrm{C}-\mathrm{NMR}\left(50 \mathrm{MHz}, \mathrm{CDCl}_{3}\right): \delta=3.1\left(\mathrm{CH}_{2}\right) ; 7.6\left(\mathrm{CH}_{3}\right) ; 21.3\left(\mathrm{CH}_{3}\right) ; 60.8\left(\mathrm{CH}_{2}\right) ; 61.7\left(\mathrm{CH}_{2}\right)$; $136.5(\mathrm{Cq}) ; 143.0(\mathrm{CH}) ; 171.5(\mathrm{Cq})$. Anal. Calcd. for $\mathrm{C}_{12} \mathrm{H}_{24} \mathrm{O}_{3} \mathrm{Si}$ (244.28): C 58.97, $\mathrm{H}$ 9.90. Found: C 59.03, H 9.82.

(E)-4-Acetoxy-1-bromo-3-triethylsilyl-but-2-ene (5'). $\mathrm{CBr}_{4}(984 \mathrm{mg}, 2.97 \mathrm{mmol}, 1.15$ equiv.)

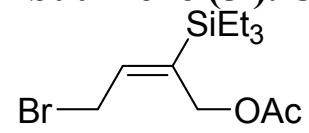

was added at $0^{\circ} \mathrm{C}$ to a solution of the allylic alcohol $5(630 \mathrm{mg}, 2.58 \mathrm{mmol})$ in $\mathrm{CH}_{2} \mathrm{Cl}_{2}(5 \mathrm{~mL})$. Then a solution of triphenylphosphine ( $880 \mathrm{mg}, 3.35 \mathrm{mmol}, 1.3$ equiv.) in $\mathrm{CH}_{2} \mathrm{Cl}_{2}(5 \mathrm{~mL})$ was transferred into the reaction mixture. After $30 \mathrm{~min}$, the mixture was concentrated under vacuum to afford $\mathbf{5}^{\prime}$ as a colorless oil. The crude product was sufficiently clean to be used directly in the next step. $\mathrm{R}_{\mathrm{f}}$ : 0.7 (PE/EA 8/2); IR: 2954, 2874, 1739, 1459, 1378, 1222, 1095, 1021, 961, 716, $673 \mathrm{~cm}^{-1} ;{ }^{1} \mathrm{H}-\mathrm{NMR}: \delta=0.65$ (q, $\left.J=8 \mathrm{~Hz}, 6 \mathrm{H}\right) ; 0.94(\mathrm{t}, J=8 \mathrm{~Hz}, 9 \mathrm{H}) ; 2.07(\mathrm{~s}, 3 \mathrm{H}) ; 4.08(\mathrm{~d}, J=8$ $\mathrm{Hz}, 2 \mathrm{H}), 4.78(\mathrm{~s}, 2 \mathrm{H}) ; 6.15(\mathrm{t}, J=8 \mathrm{~Hz}, 1 \mathrm{H})$. Anal. Calcd. for $\mathrm{C}_{12} \mathrm{H}_{23} \mathrm{O}_{2} \mathrm{BrSi}$ (307.30): C 46.90, $\mathrm{H}$ 7.54; Found: C 48.28, H 7.86.

(E)-[(4-Acetoxy-3-triethylsilyl-but-2-enyl)-(toluene-4-sulfonyl)-amino]-acetic acid methyl ester (6). To a cold $\left(0^{\circ} \mathrm{C}\right)$ solution of $5^{\prime}$ in dry THF $(8 \mathrm{~mL})$ was added a preformed solution of

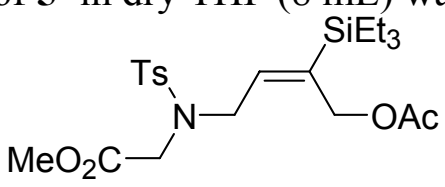

the potassium salt of N-tosyl methyl glycinate (690 mg, $2.84 \mathrm{mmol}, 1.1$ equiv.) in THF (5 mL). The solution was stirred $2 \mathrm{~h}$ at $0^{\circ} \mathrm{C}$, then quenched with a saturated solution of $\mathrm{NH}_{4} \mathrm{Cl}$. The aqueous phase was extracted twice with $\mathrm{Et}_{2} \mathrm{O}$. The combined organic layers were washed with brine, dried over $\mathrm{MgSO}_{4}$ and concentrated under reduced pressure. The crude product was purified on a silica gel column (PE/EA 8/2) to afford 6 (856 mg, $1.82 \mathrm{mmol}, 71 \%$ yield over 2 steps) as a colorless oil. $\mathrm{R}_{\mathrm{f}}=0.2$ (PE/EA 8/2); IR: 2952, 2875, 1740, 1437, 1340, 1226, 1160, 1100, 1000, 930, 720, $655 \mathrm{~cm}^{-1} ;{ }^{1} \mathrm{H}-\mathrm{NMR}\left(400 \mathrm{MHz}, \mathrm{CDCl}_{3}\right): \delta=0.57$ (q, $\left.J=7.8 \mathrm{~Hz}, 6 \mathrm{H}\right) ; 0.87$ $(\mathrm{t}, J=7.8 \mathrm{~Hz}, 9 \mathrm{H}) ; 2.00(\mathrm{~s}, 3 \mathrm{H}) ; 2.44(\mathrm{~s}, 3 \mathrm{H}) ; 3.64(\mathrm{~s}, 3 \mathrm{H}) ; 4.01(\mathrm{~s}, 2 \mathrm{H}) ; 4.08(\mathrm{~d}, J=6.8 \mathrm{~Hz}, 2 \mathrm{H})$; $4.58(\mathrm{~s}, 2 \mathrm{H}) ; 5.72(\mathrm{t}, J=6.8 \mathrm{~Hz}, 1 \mathrm{H}) ; 7.31(\mathrm{~d}, J=8.0 \mathrm{~Hz}, 2 \mathrm{H}) ; 7.74(\mathrm{~d}, J=8.0 \mathrm{~Hz}, 2 \mathrm{H}) ;{ }^{13} \mathrm{C}-\mathrm{NMR}$ $\left(100 \mathrm{MHz}, \mathrm{CDCl}_{3}\right): \delta=2.8\left(\mathrm{CH}_{2}\right) ; 7.2\left(\mathrm{CH}_{3}\right) ; 20.9\left(\mathrm{CH}_{3}\right) ; 21.5\left(\mathrm{CH}_{3}\right) ; 45.9\left(\mathrm{CH}_{2}\right) ; 47.4\left(\mathrm{CH}_{2}\right)$; 
$52.1\left(\mathrm{CH}_{3}\right) ; 61.9\left(\mathrm{CH}_{2}\right) ; 127.4(\mathrm{CH}) ; 129.7(\mathrm{CH}) ; 136.8(\mathrm{Cq}) ; 138.8(\mathrm{CH}) ; 142.5(\mathrm{Cq}) ; 143.6$ $(\mathrm{Cq}) ; 169.6(\mathrm{Cq}) ; 171.1(\mathrm{Cq})$; Anal. Calcd. for $\mathrm{C}_{22} \mathrm{H}_{35} \mathrm{NO}_{6} \mathrm{SSi}$ (469.67): C 56.26, H 7.51, N 2.98; Found: C 56.09, H 7.60, N 3.11.

(E)-[(4-Hydroxy-2-triethylsilyl-but-2-enyl)-(toluene-4-sulfonyl)-amino]-acetic acid methyl ester (7). Potassium carbonate $(531 \mathrm{mg}, 3.85 \mathrm{mmol}, 0.2$ equiv.) was added to a solution of the

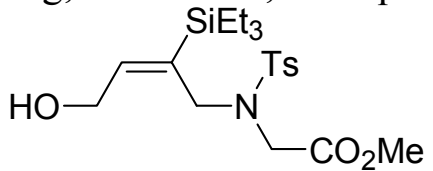

allylic acetate $4(9.05 \mathrm{~g}, 19.25 \mathrm{mmol})$ in $\mathrm{MeOH}(50 \mathrm{~mL})$. The resulting suspension was stirred 16 $\mathrm{h}$ at room temperature. The methanol was removed under reduced pressure and a mixture of water/ $\mathrm{Et}_{2} \mathrm{O}(1 / 1)$ was added. After extraction with $\mathrm{Et}_{2} \mathrm{O}$, the organic layers were washed with brine, dried over $\mathrm{MgSO}_{4}$ and concentrated. The oil was purified on a silica gel column in order to afford 7 (7.42 g, $17.36 \mathrm{mmol}, 89 \%$ yield) as a colorless oil. $\mathrm{R}_{\mathrm{f}}=0.25$ (EP/AE 6/4); IR: 3600, 2950, 2875, 1740, 1460, 1330, 1210, 1160, 1100, 1000, 914, 720, $650 \mathrm{~cm}^{-1}$. ${ }^{1} \mathrm{H}-\mathrm{NMR}(400 \mathrm{MHz}$, $\left.\mathrm{CDCl}_{3}\right): \delta=0.66(\mathrm{q}, J=7.8 \mathrm{~Hz}, 6 \mathrm{H}) ; 0.92(\mathrm{t}, J=7.8 \mathrm{~Hz}, 9 \mathrm{H}) ; 2.45(\mathrm{~s}, 3 \mathrm{H}) ; 3.52$ (s, 3H); 3.93 (s, $2 \mathrm{H}) ; 4.07(\mathrm{~s}, 2 \mathrm{H}) ; 4.22(\mathrm{~d}, J=6.3 \mathrm{~Hz}, 2 \mathrm{H}) ; 6.21(\mathrm{t}, J=6.3 \mathrm{~Hz}, 1 \mathrm{H}) ; 7.33(\mathrm{~d}, J=8.1 \mathrm{~Hz}, 2 \mathrm{H}) ; 7.70$ $(\mathrm{d}, J=8.1 \mathrm{~Hz}, 2 \mathrm{H}) ;{ }^{13} \mathrm{C}-\mathrm{NMR}\left(100 \mathrm{MHz}, \mathrm{CDCl}_{3}\right): \delta=2.6\left(\mathrm{CH}_{2}\right) ; 7.4\left(\mathrm{CH}_{3}\right) ; 21.6\left(\mathrm{CH}_{3}\right) ; 45.7$ $\left(\mathrm{CH}_{2}\right) ; 46.0\left(\mathrm{CH}_{2}\right) ; 52.0\left(\mathrm{CH}_{3}\right) ; 59.0\left(\mathrm{CH}_{2}\right) ; 127.5(\mathrm{CH}) ; 129.5(\mathrm{CH}) ; 133.6(\mathrm{Cq}) ; 135.9(\mathrm{Cq})$; $143.6(\mathrm{Cq}) ; 147.5(\mathrm{CH}) ; 169.6(\mathrm{Cq})$. Anal. Calcd. for $\mathrm{C}_{20} \mathrm{H}_{33} \mathrm{NO}_{5} \mathrm{SSi}(427.63)$ : C 56.17, $\mathrm{H} 7.78$, N 3.28; Found: C 57.66, H 8.08, N 2.71.

(E)-[(4-Hydroxy-3-triethylsilyl-but-2-enyl)-(toluene-4-sulfonyl)-amino]- acetic acid methyl ester (8). Following the same method, from the acetate $6(856 \mathrm{mg}, 1.82 \mathrm{mmol})$, the alcohol 8

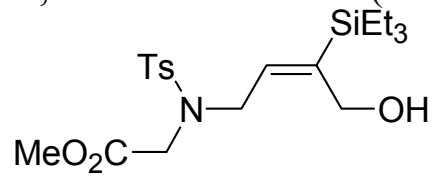

was obtained as a colorless oil (620 mg, $1.45 \mathrm{mmol}, 80 \%$ yield). $\mathrm{R}_{\mathrm{f}}=0.25$ (PE/EA 7/3); IR: 3600, 2950, 2875, 1740,1430, 1340, 1210, 1155,1100, 1000, 925, 715, $655 \mathrm{~cm}^{-1}$; ${ }^{1} \mathrm{H}-\mathrm{NMR}(400 \mathrm{MHz}$, $\left.\mathrm{CDCl}_{3}\right): \delta=0.58(\mathrm{q}, J=8.0 \mathrm{~Hz}, 6 \mathrm{H}) ; 0.89(\mathrm{t}, J=8.09 \mathrm{H}) ; 1.27(\mathrm{~s}, 1 \mathrm{H}) ; 2.44(\mathrm{~s}, 3 \mathrm{H}) ; 3.65(\mathrm{~s}, 3 \mathrm{H}) ;$ $4.04(\mathrm{~s}, 2 \mathrm{H}) ; 4.08(\mathrm{~d}, J=6.8 \mathrm{~Hz}, 2 \mathrm{H}) ; 4.17(\mathrm{~s}, 2 \mathrm{H}) ; 5.66(\mathrm{t}, J=6.8 \mathrm{~Hz}, 1 \mathrm{H}) ; 7.32$ (d, $J=8.3 \mathrm{~Hz}$, $2 \mathrm{H}) ; 7.74(\mathrm{~d}, J=8.3 \mathrm{~Hz}, 2 \mathrm{H}) ;{ }^{13} \mathrm{C}-\mathrm{NMR}\left(100 \mathrm{MHz}, \mathrm{CDCl}_{3}\right): \delta=2.8\left(\mathrm{CH}_{2}\right) ; 7.3\left(\mathrm{CH}_{3}\right) ; 21.4$ $\left(\mathrm{CH}_{3}\right) ; 45.6\left(\mathrm{CH}_{2}\right) ; 47.5\left(\mathrm{CH}_{2}\right) ; 52.2\left(\mathrm{CH}_{3}\right) ; 59.8\left(\mathrm{CH}_{2}\right) ; 127.3(\mathrm{CH}) ; 129.5(\mathrm{CH}) ; 136.7(\mathrm{CH})$; $136.5(\mathrm{Cq}) ; 143.5(\mathrm{Cq}) ; 144.2(\mathrm{Cq}) ; 169.5(\mathrm{Cq})$. Anal. Calcd. for $\mathrm{C}_{20} \mathrm{H}_{33} \mathrm{NO}_{5} \mathrm{SSi}$ (427.63): C 56.17, H 7.78, N 3.28; Found: C 55.99, H 7.90, N 3.35.

trans-[[(4-Hydroxymethyl)-(2-triethylsily)-oxiranylmethyl]-(toluene-4-sulfonyl)-amino]acetic acid methyl ester (9). To a solution of the allylic alcohol 7 (5 g, $11.71 \mathrm{mmol})$ in $\mathrm{CH}_{2} \mathrm{Cl}_{2}$

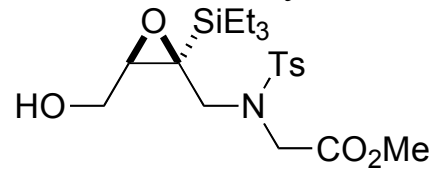

(40 mL) was added at $0^{\circ} \mathrm{C}$ the $m$-CPBA $\left(5.77 \mathrm{~g}, 23.42 \mathrm{mmol}, 70 \% m\right.$-CPBA in $\mathrm{H}_{2} \mathrm{O}, 2$ equiv.). The resulting suspension was stirred at room temperature for $14 \mathrm{~h}$. The reaction mixture was 
treated with aqueous $\mathrm{NaOH}(1 M)$. The organic layer was separated and the aqueous phase extracted with $\mathrm{CH}_{2} \mathrm{Cl}_{2}$ (3 times). The combined organic layers were washed with brine, dried over $\mathrm{MgSO}_{4}$ and evaporated under reduced pressure. The crude product was purified on a silica gel column in order to afford $9\left(4.10 \mathrm{~g}, 9.25 \mathrm{mmol}, 79 \%\right.$ yield) as a colorless oil. $\mathrm{R}_{\mathrm{f}}=0.2$ (PE/EA 7/3); IR: 3600, 2950, 2875, 1740, 1437, 1340, 1157, 1100, 1000, $720 \mathrm{~cm}^{-1}$; ${ }^{1} \mathrm{H}-\mathrm{NMR}$ (400 MHz, $\left.\mathrm{CDCl}_{3}\right): \delta=0.78(\mathrm{q}, J=8.0 \mathrm{~Hz}, 6 \mathrm{H}) ; 1.01(\mathrm{t}, J=8.0 \mathrm{~Hz}, 9 \mathrm{H}) ; 2.45(\mathrm{~s}, 3 \mathrm{H}) ; 3.07(\mathrm{t}, J=5.4 \mathrm{~Hz}, 1 \mathrm{H})$; $3.46(\mathrm{~d}, J=14.3 \mathrm{~Hz}, 1 \mathrm{H}) ; 3.47(\mathrm{~s}, 3 \mathrm{H}) ; 3.79$ (d, $J=5.4 \mathrm{~Hz}, 2 \mathrm{H}) ; 3.90(\mathrm{~d}, J=14.3 \mathrm{~Hz}, 1 \mathrm{H}) ; 4.17$ $(\mathrm{d}, J=18.4 \mathrm{~Hz}, 1 \mathrm{H}) ; 4.35(\mathrm{~d}, J=18.4 \mathrm{~Hz}, 1 \mathrm{H}) ; 7.31(\mathrm{~d}, J=8.1 \mathrm{~Hz}, 2 \mathrm{H}) ; 7.66(\mathrm{~d}, J=8.1 \mathrm{~Hz}, 2 \mathrm{H})$; ${ }^{13} \mathrm{C}-\mathrm{NMR}\left(100 \mathrm{MHz}, \mathrm{CDCl}_{3}\right): \delta=1.6\left(\mathrm{CH}_{2}\right) ; 7.3\left(\mathrm{CH}_{3}\right) ; 21.6\left(\mathrm{CH}_{3}\right) ; 47.46$ and $47.54\left(2 \mathrm{xCH}_{2}\right)$; $51.8\left(\mathrm{CH}_{3}\right) ; 55.8(\mathrm{Cq}) ; 58.4(\mathrm{CH}) ; 62.4\left(\mathrm{CH}_{2}\right) ; 127.4(\mathrm{CH}) ; 129.5(\mathrm{CH}) ; 136.0(\mathrm{Cq}) ; 143.6(\mathrm{Cq})$; 168.9 (Cq). Anal. Calcd. for $\mathrm{C}_{20} \mathrm{H}_{33} \mathrm{NO}_{6} \mathrm{SSi}$ (443.63): C 54.15, H 7.50, N 3.16; Found: C 53.75, H 7.59, N 2.78.

trans-[[(3-Hydroxymethyl)-(3-triethylsilyl)-oxiranylmethyl]-(toluene-4-sulfonyl)-amino]acetic acid methyl ester (10). From the allylic alcohol 8 (600 mg, $1.40 \mathrm{mmol})$, the epoxy-

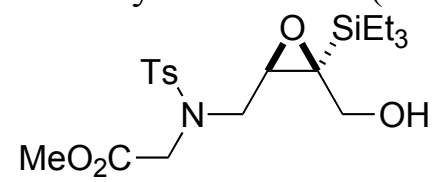

alcohol 10 (575 mg, $1.30 \mathrm{mmol}, 92 \%$ yield) was obtained as a white solid. m.p. $85^{\circ} \mathrm{C} . \mathrm{R}_{\mathrm{f}}=0.5$ (PE/EA 7/3); IR: 3600, 2950, 2875, 1740, $1330 \mathrm{~cm}^{-1} ;{ }^{1} \mathrm{H}-\mathrm{NMR}\left(400 \mathrm{MHz}, \mathrm{CDCl}_{3}\right): \delta=0.65$ (q, $J=8.0 \mathrm{~Hz}, 6 \mathrm{H}) ; 0.98(\mathrm{t}, J=8.0 \mathrm{~Hz}, 9 \mathrm{H}) ; 1.90(\mathrm{~s}, 1 \mathrm{H}) ; 2.45(\mathrm{~s}, 3 \mathrm{H}) ; 3.07(\mathrm{dd}, J=6.5$ and $4.5 \mathrm{~Hz}$, $1 \mathrm{H}) ; 3.33(\mathrm{dd}, J=15.4$ and $6.5 \mathrm{~Hz}, 1 \mathrm{H}) ; 3.66(\mathrm{~s}, 3 \mathrm{H}) ; 3.72(\mathrm{~s}, 2 \mathrm{H}) ; 3.80$ (dd, $J=15.4$ and $4.5 \mathrm{~Hz}$, $1 \mathrm{H}) ; 4.21(\mathrm{~s}, 2 \mathrm{H}) ; 7.33(\mathrm{~d}, J=8.5 \mathrm{~Hz}, 2 \mathrm{H}) ; 7.74(\mathrm{~d}, J=8.5 \mathrm{~Hz}, 2 \mathrm{H}) ;{ }^{13} \mathrm{C}-\mathrm{NMR}(100 \mathrm{MHz}$, $\left.\mathrm{CDCl}_{3}\right): \delta=1.8\left(\mathrm{CH}_{2}\right) ; 7.4\left(\mathrm{CH}_{3}\right) ; 21.7\left(\mathrm{CH}_{3}\right) ; 47.3\left(\mathrm{CH}_{2}\right) ; 49.1\left(\mathrm{CH}_{2}\right) ; 52.4\left(\mathrm{CH}_{3}\right) ; 56.4(\mathrm{Cq})$; $58.1(\mathrm{CH}) ; 62.5\left(\mathrm{CH}_{2}\right) ; 127.5(\mathrm{CH}) ; 129.9(\mathrm{CH}) ; 136.5(\mathrm{CH}) ; 144.0(\mathrm{Cq}) ; 169.4(\mathrm{Cq})$. Anal. Calcd. for $\mathrm{C}_{20} \mathrm{H}_{33} \mathrm{NO}_{6} \mathrm{SSi}$ (443.63): C 54.15, H 7.50, N 3.16. Found: C 53.98, H 7.61, N 3.22.

trans-[[3-Formyl-(2-triethylsilyl)-oxiranylmethyl]-(toluene-4-sulfonyl)-amino]-acetic acid methyl ester (1). A solution of IBX (2.19 g, $7.82 \mathrm{mmol}, 1.1$ equiv.) in DMSO (20 mL) was

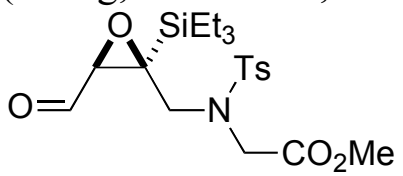

added to a solution of the epoxy-alcohol 9 (3.15 g, $7.11 \mathrm{mmol})$ in DMSO (25 mL). The resulting mixture was stirred for $3 \mathrm{~h}$ at room temperature, then cooled to $0^{\circ} \mathrm{C}$, and aqueous $\mathrm{NaHCO}_{3}(40$ $\mathrm{mL}$ ) was added. The solution was filtered to remove the white precipitate, and extracted with AcOEt. The organic layer was then washed with brine and dried over $\mathrm{MgSO}_{4}$. The crude material was purified by flash chromatography on silica gel (PE/EA 75/25) to afford 1 (2.1 g, $4.76 \mathrm{mmol}, 68 \%$ yield) as a colorless oil. $\mathrm{R}_{\mathrm{f}}=0.3(\mathrm{PE} / \mathrm{EA} 75 / 25)$; IR: $3400,2915,2875,1747$, 1435, 1337, 1160, 1010, 950, $700 \mathrm{~cm}^{-1} ;{ }^{1} \mathrm{H}-\mathrm{NMR}\left(400 \mathrm{MHz}, \mathrm{CDCl}_{3}\right): \delta=0.75$ (q, $J=7.6 \mathrm{~Hz}$, $6 \mathrm{H}) ; 1.01(\mathrm{t}, J=7.6 \mathrm{~Hz}, 9 \mathrm{H}) ; 2.42(\mathrm{~s}, 3 \mathrm{H}) ; 3.22(\mathrm{~d}, J=4.6 \mathrm{~Hz}, 1 \mathrm{H}) ; 3.46(\mathrm{~s}, 3 \mathrm{H}) ; 3.61$ (d, $J=14.4$ $\mathrm{Hz}, 1 \mathrm{H}) ; 3.90$ (d, $J=14.4 \mathrm{~Hz}, 1 \mathrm{H}) ; 4.17$ (d, $J=18.6 \mathrm{~Hz}, 1 \mathrm{H}) ; 4.35$ (d, $J=18.6 \mathrm{~Hz}, 1 \mathrm{H}) ; 7.29$ (d, $J=8.0 \mathrm{~Hz}, 2 \mathrm{H}) ; 7.61(\mathrm{~d}, J=8.0 \mathrm{~Hz}, 2 \mathrm{H}) ; 9.49(\mathrm{~d}, J=4.6 \mathrm{~Hz}, 2 \mathrm{H}) ;{ }^{13} \mathrm{C}-\mathrm{NMR}\left(100 \mathrm{MHz}, \mathrm{CDCl}_{3}\right)$ : 
$\delta=1.5\left(\mathrm{CH}_{2}\right) ; 7.4\left(\mathrm{CH}_{3}\right) ; 21.6\left(\mathrm{CH}_{3}\right) ; 46.9$ and $47.6\left(2 \mathrm{CH}_{2}\right) ; 52.0\left(\mathrm{CH}_{3}\right) ; 59.5(\mathrm{CH}) ; 61.3(\mathrm{Cq})$; $127.4(\mathrm{CH}) ; 129.6(\mathrm{CH}) ; 135.7(\mathrm{Cq}) ; 144.0(\mathrm{Cq}) ; 168.2(\mathrm{Cq}) ; 199.2(\mathrm{Cq})$. Anal. Calcd. for $\mathrm{C}_{20} \mathrm{H}_{31} \mathrm{NO}_{6} \mathrm{SSi}$ (441.62): C 54.40, H 7.08, N 3.17. Found: C 54.71, H 7.32, N 2.95.

trans-[[3-Formyl-(3-triethylsilyl)-oxiranylmethyl]-(toluene-4-sulfonyl)-amino]-acetic acid methyl ester (2). From the epoxy-alcohol 10 (540 mg, $1.22 \mathrm{mmol})$, the aldehyde 2 (368 $\mathrm{mg}, 0.83$

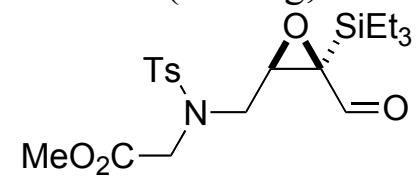

mmol) was isolated in $68 \%$ yield. $\mathrm{mp} 82^{\circ} \mathrm{C} . \mathrm{R}_{\mathrm{f}}=0.5$ (PE/EA 7/3); IR: 3600, 2950, 2875, 1740, $1330 \mathrm{~cm}^{-1} ;{ }^{1} \mathrm{H}-\mathrm{NMR}\left(400 \mathrm{MHz}, \mathrm{CDCl}_{3}\right): \delta=0.67$ (q, $\left.J=7.6 \mathrm{~Hz}, 6 \mathrm{H}\right) ; 0.97(\mathrm{t}, J=7.6 \mathrm{~Hz}, 9 \mathrm{H})$; $2.44(\mathrm{~s}, 3 \mathrm{H}) ; 3.08(\mathrm{dd}, J=15.4$ and $8 \mathrm{~Hz}, 1 \mathrm{H}) ; 3.66(\mathrm{~s}, 3 \mathrm{H}) ; 3.77(\mathrm{dd}, J=15.4$ and $3 \mathrm{~Hz}, 1 \mathrm{H})$; $4.18(\mathrm{~d}, J=18.3 \mathrm{~Hz}, 1 \mathrm{H}) ; 4.24(\mathrm{~d}, J=18.3 \mathrm{~Hz}, 1 \mathrm{H}) ; 7.32(\mathrm{~d}, J=8.3 \mathrm{~Hz}, 2 \mathrm{H}) ; 7.70$ (d, $J=8.3 \mathrm{~Hz}$, $2 \mathrm{H}) ; 9.51(\mathrm{~s}, 1 \mathrm{H}) ;{ }^{13} \mathrm{C}-\mathrm{NMR}\left(100 \mathrm{MHz}, \mathrm{CDCl}_{3}\right): \delta=1.7\left(\mathrm{CH}_{2}\right) ; 7.3\left(\mathrm{CH}_{3}\right) ; 21.7\left(\mathrm{CH}_{3}\right) ; 48.3$ and $48.9\left(2 \mathrm{CH}_{2}\right) ; 52.4\left(\mathrm{CH}_{3}\right) ; 60.4(\mathrm{CH}) ; 60.8(\mathrm{Cq}) ; 127.5(\mathrm{CH}) ; 129.5(\mathrm{CH}) ; 136.2(\mathrm{Cq}) ; 144.1(\mathrm{Cq})$; $169.1(\mathrm{Cq}) ; 202.1(\mathrm{Cq})$. Anal. Calcd. for $\mathrm{C}_{20} \mathrm{H}_{31} \mathrm{NO}_{6} \mathrm{SSi}$ (441.62): C 54.40, H 7.08, N 3.17. Found: C 54.25, H 7.25, N 3.18.

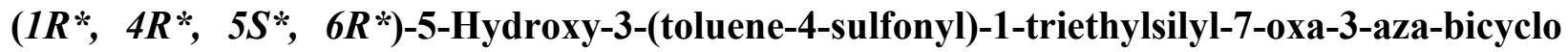
[4.1.0]heptane-4-methylcarboxylate (11a). Diazabicycloundecene $(0.2 \mathrm{~mL}, 1.4 \mathrm{mmol}$,

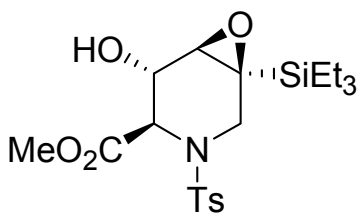

1.25 equiv.) was added to a solution of the epoxy- aldehyde 1 (496 $\mathrm{mg}, 1.12 \mathrm{mmol})$ in dry THF $(25 \mathrm{~mL})$. The solution was stirred 3 days at room temperature. Then aqueous $\mathrm{NH}_{4} \mathrm{Cl}$ was added and the aqueous layer was extracted with $\mathrm{Et}_{2} \mathrm{O}$. The organic layer was washed with brine, dried over $\mathrm{MgSO}_{4}$ and concentrated. After purification by flash chromatography, the cyclic product 11a was recrystallized from a mixture of pentane/EA (321 mg, $0.73 \mathrm{mmol}, 65 \%$ yield). $\mathrm{mp} 75^{\circ} \mathrm{C}$. $\mathrm{R}_{\mathrm{f}}=0.3\left(\mathrm{Et}_{2} \mathrm{O} / \mathrm{PE}\right.$ 6/4); IR: 3495, 2955, 2875, 1735, 1445, 1335, 1160, 1100, 1030, 905, 720, 655 $\mathrm{cm}^{-1} ;{ }^{1} \mathrm{H}-\mathrm{NMR}\left(400 \mathrm{MHz}, \mathrm{CDCl}_{3}\right): \delta=0.59(\mathrm{q}, J=7.8 \mathrm{~Hz}, 6 \mathrm{H}) ; 0.93(\mathrm{t}, J=7.8 \mathrm{~Hz}, 9 \mathrm{H}) ; 2.44$ (s, $3 \mathrm{H}) ; 3.12(\mathrm{~d}, J=2.8 \mathrm{~Hz}, 1 \mathrm{H}) ; 3.57(\mathrm{~s}, 3 \mathrm{H}) ; 3.62$ (d, $J=14.5 \mathrm{~Hz}, 1 \mathrm{H}) ; 3.89$ (d, $J=14.5 \mathrm{~Hz}, 1 \mathrm{H})$; $4.62(\mathrm{~d}, J=2.8 \mathrm{~Hz}, 1 \mathrm{H}) ; 4.83$ (pseudo dt, $J=10$ and $2.8 \mathrm{~Hz}, 1 \mathrm{H}) ; 7.32$ (d, $J=8.0 \mathrm{~Hz}, 2 \mathrm{H}) ; 7.71$ (d, $J=8.0 \mathrm{~Hz}, 2 \mathrm{H}) ;{ }^{13} \mathrm{C}-\mathrm{NMR}\left(100 \mathrm{MHz}, \mathrm{CDCl}_{3}\right): \delta=1.1\left(\mathrm{CH}_{2}\right) ; 7.2\left(\mathrm{CH}_{3}\right) ; 21.6\left(\mathrm{CH}_{3}\right) ; 41.3\left(\mathrm{CH}_{2}\right)$; $52.1\left(\mathrm{CH}_{3}\right) ; 52.4(\mathrm{Cq}) ; 55.0(\mathrm{CH}) ; 58.2(\mathrm{CH}) ; 66.9(\mathrm{CH}) ; 127.4(\mathrm{CH}) ; 129.5(\mathrm{CH}) ; 135.7(\mathrm{Cq})$; $143.8(\mathrm{Cq}) ; 167.7(\mathrm{Cq})$; Anal. Calcd. for $\mathrm{C}_{20} \mathrm{H}_{31} \mathrm{NO}_{6} \mathrm{SSi}$ (441.62): C 54.39, H 7.08, N 3.17. Found: C 54.30, H 7.09, N 3.22.

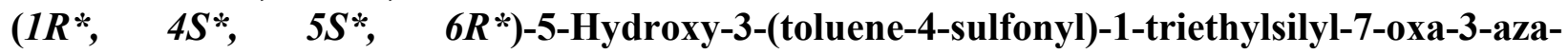
bicyclo[4.1.0]heptane-4-methylcarboxylate (11b). The other isomer, $11 \mathrm{~b}$, was isolated as a 


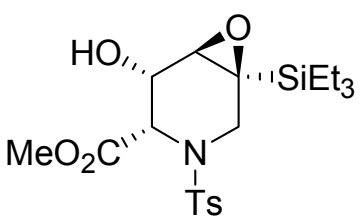

colorless oil (87 mg, $0.20 \mathrm{mmol}, 17 \%$ yield). ${ }^{1} \mathrm{H}-\mathrm{NMR}\left(400 \mathrm{MHz}, \mathrm{CDCl}_{3}\right): \delta=0.58$ (q, $J=7.3$ $\mathrm{Hz}, 6 \mathrm{H}) ; 0.93$ (t, $J=7.3 \mathrm{~Hz}, 9 \mathrm{H}) ; 2.42(\mathrm{~s}, 3 \mathrm{H}) ; 3.08(\mathrm{~s}, 1 \mathrm{H}) ; 3.41$ (d, J=14.6 Hz, 1H); 3.57 (s, $3 \mathrm{H}) ; 3.96(\mathrm{~d}, J=14.6 \mathrm{~Hz}, 1 \mathrm{H}) ; 4.69(\mathrm{~d}, J=6.8 \mathrm{~Hz}, 1 \mathrm{H}) ; 4.03(\mathrm{dd}, J=8.6$ and $6.8 \mathrm{~Hz}, 1 \mathrm{H}) ; 7.31$ (d, $J=8.3 \mathrm{~Hz}, 2 \mathrm{H}) ; 7.68(\mathrm{~d}, J=8.3 \mathrm{~Hz}, 2 \mathrm{H}) ;{ }^{13} \mathrm{C}-\mathrm{NMR}\left(100 \mathrm{MHz}, \mathrm{CDCl}_{3}\right): \delta=2.4\left(\mathrm{CH}_{2}\right) ; 6.9\left(\mathrm{CH}_{3}\right)$; $21.3\left(\mathrm{CH}_{3}\right) ; 42.9\left(\mathrm{CH}_{2}\right) ; 51.8(\mathrm{CH}) ; 52.1\left(\mathrm{CH}_{3}\right) ; 54.5(\mathrm{CH}) ; 57.1(\mathrm{CH}) ; 64.5(\mathrm{CH}) ; 127.2(\mathrm{CH})$; $129.2(\mathrm{CH}) ; 135.8(\mathrm{Cq}) ; 142.5(\mathrm{Cq}) ; 169.9(\mathrm{Cq})$.

$\left(1 R^{*}, 7 R^{*}\right)-3-($ Toluene-4-sulfonyl)-1-triethylsilyl-7-oxa-3-aza-bicyclo[4.1.0]hept-4-ene methylcarboxylate (12). From a mixture of the cyclic compounds 11a and 11b, a quantitative<smiles>CC[C@]12CN([13CH3])C(C(C)=O)=C[C@H]1O2</smiles>

dehydration took place for 11b in the presence of DBU in THF. Pure product $\mathbf{1 2}$ has been isolated in $10 \%$ yield $(21 \mathrm{mg}, 0.05 \mathrm{mmol})$ after 3 days at room temperature. $\mathrm{R}_{\mathrm{f}}=0.35\left(\mathrm{Et}_{2} \mathrm{O} / \mathrm{PE}\right.$ 6/4); IR: 2950, 2875, 1733, 1435, 1360, 1250, 1190, 1090, 1055, 1015, 945, 815, 735, 705, 685 $\mathrm{cm}^{-1} ;{ }^{1} \mathrm{H}-\mathrm{NMR}\left(400 \mathrm{MHz}, \mathrm{CDCl}_{3}\right): \delta=0.55(\mathrm{q}, J=7.8 \mathrm{~Hz}, 6 \mathrm{H}) ; 0.91(\mathrm{t}, J=7.8 \mathrm{~Hz}, 9 \mathrm{H}) ; 2.43(\mathrm{~s}$, $3 \mathrm{H}) ; 3.04(\mathrm{~d}, J=4.5 \mathrm{~Hz}, 1 \mathrm{H}) ; 3.09$ (d, $J=15.1 \mathrm{~Hz}, 1 \mathrm{H}) ; 3.83(\mathrm{~s}, 3 \mathrm{H}) ; 4.00$ (d, $J=15.1 \mathrm{~Hz}, 1 \mathrm{H})$; $6.60(\mathrm{~d}, J=4.5 \mathrm{~Hz}, 1 \mathrm{H}) ; 7.30(\mathrm{~d}, J=7.8 \mathrm{~Hz}, 2 \mathrm{H}) ; 7.69(\mathrm{~d}, J=7.8 \mathrm{~Hz}, 2 \mathrm{H}) ;{ }^{13} \mathrm{C}-\mathrm{NMR}(100 \mathrm{MHz}$, $\left.\mathrm{CDCl}_{3}\right): \delta=1.3\left(\mathrm{CH}_{2}\right) ; 7.1\left(\mathrm{CH}_{3}\right) ; 21.6\left(\mathrm{CH}_{3}\right) ; 47.0\left(\mathrm{CH}_{2}\right) ; 51.9(\mathrm{Cq}) ; 52.7\left(\mathrm{CH}_{3}\right) ; 65.9(\mathrm{CH})$; $125.2(\mathrm{CH}) ; 128.2(\mathrm{CH}) ; 129.2(\mathrm{CH}) ; 132.4(\mathrm{Cq}) ; 135.1(\mathrm{Cq}) ; 143.7(\mathrm{Cq}) ; 164.5(\mathrm{Cq})$.

2-Hydroxy-4-(toluene-4-sulfonyl)-1-triethylsilyl-7-oxa-4-aza-bicyclo[4.1.0]heptane 3-methyl carboxylate (13). From the aldehyde $2(150 \mathrm{mg}, 0.29 \mathrm{mmol})$, in the presence of DBU, a

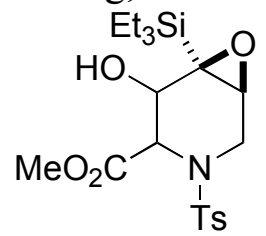

mixture of cyclic products $\mathbf{1 3}$ could be isolated. Careful purification allowed the isolation of fractions containing a mixture of two diastereomers ( $57 \mathrm{mg}, 0.13 \mathrm{mmol}, 45 \%$ yield). ${ }^{1} \mathrm{H}-\mathrm{NMR}$ $\left(400 \mathrm{MHz}, \mathrm{CDCl}_{3}\right): \delta=0.55-0.70(2 \mathrm{xq}, 2 \mathrm{x} 6 \mathrm{H}) ; 0.95(2 \mathrm{xt}, 2 \mathrm{x} 9 \mathrm{H}) ; 2.43(\mathrm{~s}, 3 \mathrm{H}, \mathrm{dia})$ ); $2.44(\mathrm{~s}, 3 \mathrm{H}$ dia2); $3.14(\mathrm{~d}, J=4 \mathrm{~Hz}, 1 \mathrm{H}$, dia1); 3.20 (d, $J=4 \mathrm{~Hz}, 1 \mathrm{H}, \operatorname{dia} 2) ; 3.46$ (s, 3H, dia2); 3.48 (s, $3 \mathrm{H}$, dia1); 3.71 (d, $J=13 \mathrm{~Hz}, 1 \mathrm{H}$, dia1); 3.82 (dd, $J=13.5$ and $4 \mathrm{~Hz}, 1 \mathrm{H}, \mathrm{dia} 2) ; 4.02$ (dd, $J=13$ and 4 $\mathrm{Hz}, 1 \mathrm{H}$, dia1); 4.13 (d, $J=13.5 \mathrm{~Hz}, 1 \mathrm{H}$, dia2); 4.54 (d, $J=2.8 \mathrm{~Hz}, 1 \mathrm{H}$, dia1); 4.60 (s, 1H, dia2); $4.63(\mathrm{~d}, J=5.8 \mathrm{~Hz}, 1 \mathrm{H}$, dia2); $4.71(\mathrm{dd}, J=11$ and $2.8 \mathrm{~Hz}, 1 \mathrm{H}$, dia1); $7.31(\mathrm{~m}, 2 \mathrm{x} 2 \mathrm{H}) ; 7.68(\mathrm{~m}$, 2x2H). ${ }^{13} \mathrm{C}-\mathrm{NMR}\left(100 \mathrm{MHz}, \mathrm{CDCl}_{3}\right): \delta=1.5\left(\mathrm{CH}_{2}\right) ; 7.3\left(\mathrm{CH}_{3}\right) ; 21.6\left(\mathrm{CH}_{3}\right) ; 39.7\left(\mathrm{CH}_{2}\right) ; 51.7$ $(\mathrm{Cq}) ; 52.4\left(\mathrm{CH}_{3}\right) ; 58.8(\mathrm{CH}) ; 69.5(\mathrm{CH}) ; 127.5(\mathrm{CH}) ; 129.5(\mathrm{CH}) ; 135.2(\mathrm{Cq}) ; 143.7(\mathrm{Cq}) ; 168.1$ $(\mathrm{Cq})$. 
$\left(1 R^{*}, 4 R^{*}, 5 S^{*}, 6 R^{*}\right)-5-($ tert-Butyldimethyl-silyloxy)-3-(toluene-4-sulfonyl)-1-triethylsilyl-7oxa-3-aza-bicyclo[4.1.0)heptane 4-methylcarboxylate (14). Dimethyl tert-butylsilyl chloride

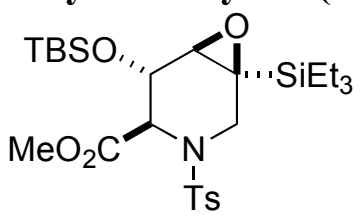

(143.3 mg, $0.95 \mathrm{mmol}, 1.5$ equiv.) and imidazole ( $86 \mathrm{mg}, 1.27 \mathrm{mmol}, 2.0$ equiv.) were added to a solution of alcohol 11a $(280 \mathrm{mg}, 0.63 \mathrm{mmol})$ in dry DMF $(1 \mathrm{~mL})$ at $0^{\circ} \mathrm{C}$. The mixture was stirred for $4 \mathrm{~h}$ and reaction stopped by adding a solution of aqueous $\mathrm{NH}_{4} \mathrm{Cl}$, and extracted with $\mathrm{Et}_{2} \mathrm{O}$. The organic combined layer were washed with brine, dried over $\mathrm{MgSO}_{4}$ and concentrated under vacuum. The crude was purified by flash chromatography (PE/EA 9/1) to afford 14 (318 $\mathrm{mg}$, $0.57 \mathrm{mmol}, 90 \%$ yield) as a colorless oil. $\mathrm{R}_{\mathrm{f}}=0.6$ (PE/EA 7/3); IR: 2955, 2875, 1735, 1460, 1335, 1255, 1160, 1100, 1035, 1000, 835, 775, 730, $655 \mathrm{~cm}^{-1} ;{ }^{1} \mathrm{H}-\mathrm{NMR}\left(400 \mathrm{MHz}, \mathrm{CDCl}_{3}\right): \delta=$ 0.20 (s, 3H); 0.23 (s, 3H); 0.49 (q, $J=8 \mathrm{~Hz}, 6 \mathrm{H}) ; 0.90$ (m, 18H); 2.41 (s, 3H); 2.95 (s, 1H); 3.50 $(\mathrm{m}, 2 \mathrm{H}) ; 3.64$ (s, 3H); 4.62 (d, $J=2 \mathrm{~Hz}, 1 \mathrm{H}) ; 4.90$ (d, $J=2 \mathrm{~Hz}, 1 \mathrm{H}) ; 7.27$ (d, J=8 Hz, 2H); 7.78 $(\mathrm{d}, J=8 \mathrm{~Hz}, 2 \mathrm{H}) ;{ }^{13} \mathrm{C}-\mathrm{NMR}\left(100 \mathrm{MHz}, \mathrm{CDCl}_{3}\right): \delta=-3.6$ et $-4.9\left(2 \mathrm{CH}_{3}\right) ; 1.0\left(\mathrm{CH}_{2}\right) ; 7.0\left(\mathrm{CH}_{3}\right)$; $14.2(\mathrm{Cq}) ; 21.5\left(\mathrm{CH}_{3}\right) ; 25.7\left(\mathrm{CH}_{3}\right) ; 41.1\left(\mathrm{CH}_{2}\right) ; 51.9(\mathrm{Cq}) ; 52.2\left(\mathrm{CH}_{3}\right) ; 55.3\left(\mathrm{C}_{\mathrm{b}}\right), 58.1(\mathrm{CH})$; $67.7(\mathrm{CH}) ; 127.7(\mathrm{CH}) ; 129.3(\mathrm{CH}) ; 136.6(\mathrm{Cq}) ; 143.4(\mathrm{Cq}) ; 168.8\left(\mathrm{C}_{\mathrm{q}}\right)$. HRMS Calc for $\mathrm{C}_{26} \mathrm{H}_{45} \mathrm{NO}_{6} \mathrm{NaSi}_{2} \mathrm{~S}:$ 578.2407. Found: 578.2404. Anal. Calcd: C 56.18, H 8.16, N 2.52. Found: C $56.69, \mathrm{H} 8.42, \mathrm{~N} 2.61$.

$\left(1 R *, 4 S^{*}, 5 S^{*}, 6 R^{*}\right)-5$-[(tert-Butyldimethyl-silyloxy)-3-(toluene-4-sulfonyl)-1-triethylsilyl-7oxa-3-aza-bicyclo[4.1.0]hept-4-yl]-methanol (15). To the ester 14 (50 mg, $0.09 \mathrm{mmol})$ diluted

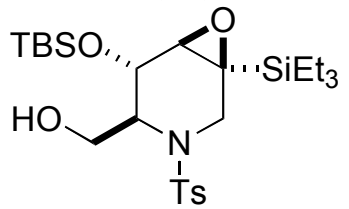

in $\mathrm{Et}_{2} \mathrm{O}(0.5 \mathrm{~mL})$ was added at $0^{\circ} \mathrm{C}$, a solution of $\mathrm{LiAlH}_{4}(10.5 \mathrm{mg}, 0.27 \mathrm{mmol}, 3$ equiv. $)$ in $\mathrm{Et}_{2} \mathrm{O}$ $(1 \mathrm{~mL})$. After $1 \mathrm{~h}$ at $0^{\circ} \mathrm{C}$, the mixture was quenched with a saturated solution of $\mathrm{Na}_{2} \mathrm{SO}_{4}$. The white-grey precipitate was filtered over Celite and washed with $\mathrm{Et}_{2} \mathrm{O}$. The organic layer was dried with $\mathrm{Na}_{2} \mathrm{SO}_{4}$, filtered and concentrated under vacuum. The alcohol 15 was isolated by flash chromatography as a colorless oil (40 mg, $0.076 \mathrm{mmol}, 84 \%$ yield). $\mathrm{R}_{\mathrm{f}}=0.6$ (PE/EA 7/3); IR: 3600, 2955, 2875, 1335, 1255, 1160, 1035, 1000, 835, 775, 730, $655 \mathrm{~cm}^{-1}$; ${ }^{1} \mathrm{H}-\mathrm{NMR}$ (400 MHz, $\left.\mathrm{CDCl}_{3}\right): \delta=0.10$ and $0.12(2 \mathrm{~s}, 6 \mathrm{H}) ; 0.46(\mathrm{q}, J=7.7 \mathrm{~Hz}, 6 \mathrm{H}) ; 0.86(\mathrm{t}, J=7.7 \mathrm{~Hz}, 9 \mathrm{H}) ; 0.89$ (s, $9 \mathrm{H}) ; 2.41(\mathrm{~s}, 3 \mathrm{H}) ; 2.96(\mathrm{~d}, J=2 \mathrm{~Hz}, 1 \mathrm{H}) ; 3.53(\mathrm{~d}, J=14.9 \mathrm{~Hz}, 1 \mathrm{H}) ; 3.67(\mathrm{~d}, J=14.9 \mathrm{~Hz}, 1 \mathrm{H})$; $3.88(\mathrm{dd}, J=11.5$ and $7 \mathrm{~Hz}, 1 \mathrm{H}) ; 3.98(\mathrm{td}, J=6.5$ and $1.2 \mathrm{~Hz}, 1 \mathrm{H}) ; 4.32(\mathrm{t}, J=2 \mathrm{~Hz}, 1 \mathrm{H}) ; 7.26(\mathrm{~d}$, $J=8.3 \mathrm{~Hz}, 2 \mathrm{H}) ; 7.82(\mathrm{~d}, J=8.3 \mathrm{~Hz}, 2 \mathrm{H}) ;{ }^{13} \mathrm{C}-\mathrm{NMR}\left(100 \mathrm{MHz}, \mathrm{CDCl}_{3}\right): \delta=-3.6$ et $-4.9\left(\mathrm{CH}_{3}\right)$; $1.0\left(\mathrm{CH}_{2}\right) ; 7.1\left(\mathrm{CH}_{3}\right) ; 18.2(\mathrm{Cq}) ; 21.5\left(\mathrm{CH}_{3}\right) ; 25.8\left(\mathrm{CH}_{3}\right) ; 41.9\left(\mathrm{CH}_{2}\right) ; 52.2(\mathrm{Cq}) ; 55.7(\mathrm{CH}), 60.7$ $(\mathrm{CH}) ; 61.6\left(\mathrm{CH}_{2}\right) ; 66.4(\mathrm{CH}) ; 127.9(\mathrm{CH}) ; 129.9(\mathrm{CH}) ; 136.7(\mathrm{Cq}) ; 143.4(\mathrm{Cq})$. 
$\left(2 S^{*}, 3 S^{*}, 4 R^{*}, 5 S^{*}\right)-3-($ tert-Butyldimethyl-silyloxy)-2-hydroxymethyl-1-(toluene-4-sulfonyl) -5-triethylsilyl-piperidin-4-ol (16). $\mathrm{LiAlH}_{4}(90 \mathrm{mg}, 2.30 \mathrm{mmol}, 4$ equiv.) was added to<smiles>CC[SiH2]C1CN([13CH3])[C@@H](CO)[C@@H](O[AsH3])[C@H]1O</smiles>

a solution of $14(318 \mathrm{mg}, 0.58 \mathrm{mmol})$ in $\mathrm{Et}_{2} \mathrm{O}$ at $0^{\circ} \mathrm{C}$. The mixture was stirred at room temperature during $22 \mathrm{~h}$ then quenched with a saturated solution of $\mathrm{Na}_{2} \mathrm{SO}_{4}$, filtered and concentrated under vacuum. The crude product was purified by flash chromatography (PE/EA $7 / 3)$ to afford $16(0.172 \mathrm{~g}, 0.31 \mathrm{mmol}, 54 \%$ yield $)$ which slowly crystallized. m.p. $114^{\circ} \mathrm{C} .{ }^{1} \mathrm{H}-$ $\operatorname{NMR}\left(400 \mathrm{MHz}, \mathrm{CDCl}_{3}\right): \delta=0.08(\mathrm{~s}, 3 \mathrm{H}), 0.12(\mathrm{~s}, 3 \mathrm{H}), 0.51(\mathrm{~m}, 6 \mathrm{H}), 0.84(\mathrm{t}, J=7.8 \mathrm{~Hz}, 9 \mathrm{H})$, $0.91(\mathrm{~s}, 9 \mathrm{H}), 1.43(\mathrm{ddd}, J=13.4,4.0$ and $1.8 \mathrm{~Hz}, 1 \mathrm{H}), 2.42(\mathrm{~s}, 3 \mathrm{H}), 3.30(\mathrm{dd}, J=14$ and $4 \mathrm{~Hz}$, $1 \mathrm{H}), 3.53(\mathrm{~d}, J=14 \mathrm{~Hz}, 1 \mathrm{H}), 3.69(\mathrm{~s}, 1 \mathrm{H}), 3.83(\mathrm{dd}, J=3.3$ and $1.5 \mathrm{~Hz}, 1 \mathrm{H}), 3.95(\mathrm{dd}, J=11.1$ and $3.6 \mathrm{~Hz}, 1 \mathrm{H}), 4.02(\mathrm{dd}, J=11.1$ and $6.3 \mathrm{~Hz}, 1 \mathrm{H}), 4.08(\mathrm{~m}, 1 \mathrm{H}), 7.24(\mathrm{~d}, J=8.4 \mathrm{~Hz}, 2 \mathrm{H}), 7.83(\mathrm{~d}$, $J=8.1 \mathrm{~Hz}, 2 \mathrm{H}) ;{ }^{13} \mathrm{C}-\mathrm{NMR}:-4.98\left(\mathrm{CH}_{3}\right),-4.92\left(\mathrm{CH}_{3}\right), 2.5\left(3 \mathrm{CH}_{2}\right), 7.3\left(3 \mathrm{CH}_{3}\right), 18.1(\mathrm{Cq}), 21.1$ $\left(\mathrm{CH}_{3}\right), 21.5(\mathrm{CH}), 25.8\left(3 \mathrm{xCH}_{3}\right), 39.1\left(\mathrm{CH}_{2}\right), 60.2(\mathrm{CH}), 64.9\left(\mathrm{CH}_{2}\right), 69.0(\mathrm{CH}), 70.8(\mathrm{CH})$, $127.6(\mathrm{CH}), 129.4(\mathrm{CH}), 137.8(\mathrm{Cq}), 143.0(\mathrm{Cq})$. Anal. Calcd. For $\mathrm{C}_{25} \mathrm{H}_{47} \mathrm{NO}_{5} \mathrm{SSi}_{2}$ (529.987): C 56.67, H 8.94, N 2.64. Found: C 56.78, H 9.15, N 2.68. HRMS Calcd for $\mathrm{C}_{25} \mathrm{H}_{47} \mathrm{NO}_{5} \mathrm{SSi}_{2} \mathrm{Na}$ : 552.2595. Found 552.2611.

\section{Acknowledgements}

We gratefully acknowledge financial support from UPMC, CNRS, and Institut Universitaire de France of which M.M. is a senior member.

\section{References and Notes}

1. (a) Tsuji, J. Palladium Reagents and Catalysts; Wiley: New York, 1995. (b) Handbook of Organopalladium Chemistry for Organic Synthesis; Negishi, E., Ed.; Wiley: New York, 2002. (c) See a recent Tetrahedron Symposia-in-print. Guest Ed. Fairlamb, I. J. S., Development and application of highly active and selective palladium catalysts. Tetrahedron 2005, 61, 9657.

2. For two recent reviews on the utilization of $\mathrm{Pd} / \mathrm{C}$ in coupling reactions, see (a) Seki, M. Synthesis 2006, 18, 2975. (b) Yin, L.; Liebscher, J. Chem. Rev. 2007, 107, 133. (c) See also Astruc, D. Inorg. Chem. 2007, 46, 1884.

3. For a review, see: Ansell, J.; Wills, M. Chem. Soc. Rev. 2002, 31, 259. 
4. More than 1000 ligands have been described to date. (a) Woska, D.; Prock, A.; Giering, W. P. Organometallics 2000, 19, 4929. (b) Trost, B. M.; Machacek, M. R.; Aponick, A. Acc. Chem. Res. 2006, 39, 747.

5. For a review on water as solvent, see: (a) Shaughnessy, K. H. Eur. J. Org. Chem. 2006, 8, 1827. For a review on catalytic reactions in ionic liquids, see: Sheldon, R. Chem. Commun. 2001, 2399.

6. (a) For a DFT study dealing with the influence of the electronic factors on the regioselectivity of palladium-catalyzed alkylations, see: Branchadell, V.; Moreno-Mañas, M.; Pajuelo, F.; Pleixats, R. Organometallics 1999, 18, 4934. (b) See also: Steinhuebel, D.; Palucki, M.; Davies, I.W. J. Org. Chem. 2006, 71, 3282.

7. (a) Hirao, T.; Enda, J.; Ohshiro, Y.; Agawa, T. Tetrahedron Lett. 1981, 22, 3079. (b) Branchadell, V.; Moreno-Mañas, M.; Pleixats, R. Organometallics 2002, 21, 2407.

8. (a) Langkopf, E.; Schinzer, D. Chem. Rev. 1995, 95, 1375. (b) Fleming, I.; Barbero, A.; Walter, D. Chem. Rev. 1997, 97, 2063. (c) Liu, D.; Kozmin, S. A. Angew. Chem. Int. Ed. 2001, 40, 4757. (d) Chabaud, L.; James, P.; Landais, Y. Eur. J. Org. Chem. 2004, 3173. (e) Fauvel, A.; Deleuze, H.; Landais, Y. Eur. J. Org. Chem. 2005, 3900.

9. (a) Büttner, M. W.; Penka, M.; Doszczak, L.; Krapft, P.; Tacke, R. Organometallics 2007, 26, 1295. (b) Tacke, R.; Schmid, T.; Merget, M. Organometallics 2005, 24, 1780. (c) Cavelier, F.; Vivet, B.; Martinez, J.; Aubry, A.; Didierjean, C.; Vicherat, A.; Marraud, M. J. Am. Chem. Soc. 2002, 124, 2917. (d) Bolm, C.; Kasyan, A.; Drauz, K.; Günther, K.; Raabe, G. Angew. Chem. Int. Ed. 2000, 39, 2288.

10. (a) Itami, K.; Nokami, T.; Yoshida, J. J. Am. Chem. Soc. 2001, 123, 5600. (b) Inami, H.; Ito, T.; Urabe, H.; Sato, F. Tetrahedron Lett. 1993, 34, 5919. (c) Olofsson, K.; Larhed, M.; Hallberg, A. J. Org. Chem. 1998, 63, 5076.

11. Ollivier, J.; Girard, N.; Salaün, J. Synlett 1999, 1539.

12. For monographs dealing with the behavior of organosilicon compounds in palladiumcatalyzed cross-coupling reactions, see (a) Hiyama, T. J. Organomet. Chem. 2002, 653, 58. (b) Nakao, Y.; Imanaka, H.; Chen, J.; Yada, A.; Hiyama, T. J. Organomet. Chem. 2007, 692, 585. (c) Denmark, S.E.; Yang, S.M. Tetrahedron 2004, 60, 9695.

13. (a)Thorimbert, S.; Malacria, M. Tetrahedron Lett. 1996, 37, 8483. (b) Commandeur, C.; Thorimbert, S.; Malacria, M. J. Org. Chem. 2003, 68, 5588. (c) For a recent application of the silicon effect in palladium-catalyzed alkylation, see: Vitale, M.; Prestat, G.; Lopes, D.; Madec, D.; Poli, G. Synlett 2006, 2231.

14. Branchadell, V.; Moreno-Mañas, M.; Pleixats, R.; Thorimbert, S.; Commandeur, C.; Boglio, C.; Malacria, M. J. Organomet. Chem. 2003, 687, 337.

15. Otha, T.; Hosokawa, T.; Murahashi, S.-I.; Miki, K.; Kasai, N. Organometallics, 1985, 4, 2080 .

16. Thorimbert, S.; Malacria, M. Tetrahedron Lett. 1998, 39, 9659.

17. (a) Humilière, D.; Thorimbert, S.; Malacria, M. Synlett 1998, 1255. (b) Thorimbert, S.; Taillier, C.; Bareyt, S.; Humilière, D.; Malacria, M. Tetrahedron Lett. 2004, 45, 9123. 
18. Boglio, C.; Stahlke, S.; Thorimbert, S.; Malacria, M. Org. Lett. 2005, 7, 4851.

19. For some reviews see: (a) Laschat, S.; Dickner, T. Synthesis 2000, 1781. (b) Weintraub, P. M.; Sabol, J. S.; Kane, J. M.; Borcherding, D. R. Tetrahedron 2003, 59, 2953. (c) Felpin, F.X.; Lebreton, J. Eur. J. Org. Chem. 2003, 3693. (d) Buffat, M. G. P. Tetrahedron 2004, 60, 1701 .

20. For recent reviews see (a) Pearson, M. S. M.; Mathé-Allainmat, M.; Fargeas, V.; Lebreton, J. Eur. J. Org. Chem. 2005, 2159. (b) Huang, P.-Q. Synlett 2006, 1133.

21. For selected approaches see (a) Sletten, E. M.; Liotta, L. J. J. Org. Chem. 2006, 71, 1335. (b) Calderón, F.; Doyagüez, E. G.; Fernández-Mayorales, A. J. Org. Chem. 2006, 71, 6258. (c) Guo, H.; O'Doherty, G. A. Org. Lett. 2006, 8, 1609. (d) Wang, R.-W.; Quing, F.-L. Org. Lett. 2005, 7, 2189. (e) Segraves, N. L.; Crews, P. J. Nat. Prod. 2005, 68, 118. (f) Goodenough, K. M.; Raubo, P.; Harrity, J. P. A. Org. Lett. 2005, 7, 2993. (g) Martìn, R.; Murruzzu, C.; Pericàs, M. A.; Riera, A. J. Org. Chem. 2005, 70, 2325. (h) Legault, C. Y.; Charette, A. B. J. Am. Chem. Soc. 2005, 127, 8966. (i) Takahata, H.; Banba, Y.; Sasatani, M.; Nemoto, H.; Kato, A.; Adachi, I. Tetrahedron 2004, 60, 8199. (j) Adelbrecht, J. C.; Craig, D.; Dymock, B. W.; Thorimbert, S. Synlett 2002, 467.

22. For related intramolecular aldolizations see: (a) Danieli, B.; Lesma, G.; Palmisano, G. Tetrahedron Lett. 1981, 22, 1827. (b) Chandrakala, P. S.; Katz, A. M.; Carell, H. L.; Sailaje, P. R.; Podile, A. R.; Nangia, A.; Desiraju, G. R. J. Chem. Soc., Perkin Trans. 1 1998, 2597. (c) Bravin, F. M.; Busnelli, G.; Colombo, M.; Gatti, F.; Manzoni, L.; Scolastico, C. Synthesis 2004, 353.

23. Seebach, D.; Hungerbuhler, E.; Naef, R.; Schnurrenberger, P.; Weidmann, B. Synthesis, 1982, 138.

24. (a) Chérest, M.; Felkin, H.; Prudent, N. Tetrahedron Lett. 1978, 18, 2199. (b) Anh, N. T.; Eisenstein, O.; Lefour, J. M.; Trân Huu Dâu, M. E. J. Am. Chem. Soc. 1973, 95, 6146. (c) Mengel, A.; Reiser, O. Chem. Rev. 1999, 99, 1191.

25. (a) Eisch, J. J.; Trainor, J. T. J. Org. Chem. 1963, 28, 2870. (b) Fristad, W. E.; Bailey, T. R.; Paquette, L. A.; Gleiter, R.; Boehm, M. C. J. Am. Chem. Soc. 1979, 101, 4420. 